\title{
Stereotactic body radiation therapy use for high risk prostate cancer in the United States
}

\author{
Sagar A. Patel $\mathbb{1}^{1}$ - Jeffrey M. Switchenko ${ }^{2}$ - Amar U. Kishan ${ }^{3}$ - Ashesh B. Jani ${ }^{1}$ - Trevor J. Royce $\mathbb{C}^{4}$ • \\ Benjamin W. Fischer-Valuck ${ }^{1}$
}

Received: 18 August 2020 / Revised: 22 October 2020 / Accepted: 30 October 2020 / Published online: 13 November 2020

(c) The Author(s), under exclusive licence to Springer Nature Limited part of Springer Nature 2020. This article is published with open access

\section{To the Editor:}

Background: Stereotactic body radiation therapy (SBRT) is increasingly utilized for localized prostate cancer $(\mathrm{PCa})$. Although its use is more widely supported for low/intermediate risk disease, the 2020 National Comprehensive Cancer Network (NCCN) guidelines now endorse its use for high risk (HR) as well. Clinicodemographic factors associated with SBRT use in HRPCa in the United States remain unknown.

Methods: Men $>40$ years with localized HRPCa (cT3-4 or Gleason $8-10$ or PSA $>20$ ) treated with external radiation between 2004 and 2016 were identified from the National Cancer Database. Overall, 1157 men were treated with SBRT and 48,498 with external beam radiation therapy (EBRT). Chi-square/ANOVA compared clinical/ demographic characteristics, Cochran-Armitage assessed utilization trends, and multivariable logistic regression (MVA) computed odds of receiving SBRT over EBRT.

Results: SBRT use for HRPCa increased over threefold from 2004 to $2016(0.8 \%$ in 2004 to $2.8 \%$ in 2016, $p<0.001)$, which was largely driven by increased

Supplementary information The online version of this article (https:// doi.org/10.1038/s41391-020-00300-5) contains supplementary material, which is available to authorized users.

Sagar A. Patel

sagar.patel@emory.edu

1 Department of Radiation Oncology, Winship Cancer Institute of Emory University, Atlanta, GA, USA

2 Department of Biostatistics and Bioinformatics, Emory University, Atlanta, GA, USA

3 Department of Radiation Oncology, University of California, Los Angeles, Los Angeles, CA, USA

4 Department of Radiation Oncology, University of North Carolina at Chapel Hill, Chapel Hill, NC, USA utilization in men not receiving concomitant androgen deprivation therapy (ADT; $0.7 \%$ in 2004 to $8.3 \%$ in 2016) and those with PSA $>20$ as the only HR factor $(1.0 \%$ in 2004 to $4.3 \%$ in 2016). The omission of concomitant ADT, clinical stage T1-2, and Gleason score 6-7 was independently associated with increased odds of SBRT receipt. In addition, medical comorbidities and longer travel distance for treatment were associated with receiving SBRT.

Conclusion: Lower clinical stage, lower Gleason score, and omission of ADT are associated with SBRT use in HRPCa. The increase in use since 2004 has dominated by men with PSA $>20$ as the only qualifying HR factor.

Ultrahypofractionation using SBRT is an advanced technique delivering large radiation doses in $\leq 5$ treatments with cost and patient convenience advantages. As treatment volumes are smaller and exclude pelvic lymph nodes, it is predominantly utilized for low/intermediate risk PCa $[1,2]$. In 2020, the NCCN added SBRT to conventional/moderate radiation regimens to the list of options for HR PCa but especially when protracted radiation courses present medical/social hardship [3]. This change occurred after the randomized HYPO-RT-PC trial [4] showed non-inferiority of ultrahypofractionation compared to conventional fractionation ( 40 treatments) for tumor control and toxicity in intermediate and HR PCa after 5-year follow-up. The change also proceeded an informative meta-analysis of 39 prospective studies which showed favorable 5- and 7-year biochemical control and low toxicity [5]. However, only $8 \%$ of men had HRPCa; furthermore, those studies that included HR men did not separately report outcomes by risk group. Other data specifically supporting SBRT for HRPCa are retrospective with majority $<5$ years follow-up [6].

While SBRT use is increasing across all PCa risk groups in the US [1], a detailed analysis of the clinicodemographic factors associated with its use in HRPCa is lacking and is the subject of this analysis.

Men $>40$ years with localized, NCCN-defined HRPCa (T3-4 or Gleason 8-10 or PSA $>20$ ) treated with external 
Table 1 Multivariable logistic regression defined adjusted odds ratios for receipt of stereotactic body radiation therapy (SBRT).

\begin{tabular}{|c|c|c|c|}
\hline Covariate & Level & $\begin{array}{l}\text { Odds ratio } \\
(95 \% \mathrm{CI})\end{array}$ & $p$ value \\
\hline \multirow[t]{2}{*}{ Age at diagnosis } & $\geq 65$ & $0.86(0.72-1.04)$ & 0.124 \\
\hline & $<65$ & - & - \\
\hline \multirow[t]{4}{*}{ Race/ethnicity } & Other & $0.94(0.64-1.37)$ & 0.741 \\
\hline & Hispanic & $0.95(0.67-1.33)$ & 0.751 \\
\hline & Black & $1.01(0.85-1.21)$ & 0.889 \\
\hline & White & - & - \\
\hline \multirow[t]{2}{*}{ Facility type } & Academic program & $2.16(1.89-2.46)$ & $<0.001$ \\
\hline & Non-academic program & - & - \\
\hline \multirow[t]{2}{*}{ Zip code median household income } & $<\$ 46,000$ & $0.50(0.42-0.59)$ & $<0.001$ \\
\hline & $\geq \$ 46,000$ & - & - \\
\hline \multirow[t]{4}{*}{ Zip code percent without high school degree } & $\geq 29 \%$ & $1.26(0.92-1.65)$ & 0.131 \\
\hline & $20-28.9 \%$ & $1.01(0.83-1.25)$ & 0.888 \\
\hline & $14-19.9 \%$ & $0.89(0.74-1.07)$ & 0.234 \\
\hline & $<14 \%$ & - & - \\
\hline \multirow[t]{6}{*}{ Insurance status } & Not insured & $0.60(0.32-1.14)$ & 0.116 \\
\hline & Private insurance/managed care & $0.67(0.42-1.07)$ & 0.093 \\
\hline & Medicaid & $0.53(0.29-0.95)$ & 0.034 \\
\hline & Medicare & $0.78(0.49-1.23)$ & 0.283 \\
\hline & Other government & $0.82(0.48-1.41)$ & 0.473 \\
\hline & Insurance status unknown & - & - \\
\hline \multirow[t]{4}{*}{ Facility location (Geographic) } & Northeast & $1.88(1.51-2.33)$ & $<0.001$ \\
\hline & South & $1.33(1.07-1.65)$ & 0.010 \\
\hline & Midwest & $1.03(0.82-1.30)$ & 0.773 \\
\hline & West & - & - \\
\hline \multirow[t]{3}{*}{ Distance to treatment Facility } & $50+$ miles & $3.66(3.00-4.46)$ & $<0.001$ \\
\hline & 25-50 miles & $1.94(1.61-2.33)$ & $<0.001$ \\
\hline & $0-25$ miles & - & - \\
\hline \multirow[t]{2}{*}{ Charlson-Deyo comorbidity score } & $1+$ & $1.23(1.04-1.46)$ & 0.014 \\
\hline & 0 & - & - \\
\hline \multirow[t]{2}{*}{ Facility location (Urban/Rural) } & Metro/urban & $2.51(1.36-4.63)$ & 0.003 \\
\hline & Rural & - & - \\
\hline \multirow[t]{2}{*}{ T-stage } & T3-4 & $0.53(0.43-0.66)$ & $<0.001$ \\
\hline & $\mathrm{T} 1-2$ & - & - \\
\hline \multirow[t]{2}{*}{ Receipt of ADT } & Yes & $0.19(0.16-0.21)$ & $<0.001$ \\
\hline & No & - & - \\
\hline \multirow[t]{2}{*}{ PSA } & $>20$ & $0.99(0.82-1.20)$ & 0.926 \\
\hline & $\leq 20$ & - & - \\
\hline \multirow[t]{2}{*}{ Gleason score } & $8-10$ & $0.56(0.46-0.68)$ & $<0.001$ \\
\hline & $6-7$ & - & - \\
\hline
\end{tabular}

Bold values indicate statistical significance $p<0.05$.

$A D T$ androgen deprivation therapy. radiation between 2004 and 2016 were identified from the National Cancer Database. SBRT was defined as $\geq 5 \mathrm{~Gy} /$ fraction and $\leq 5$ fractions, non-SBRT EBRT as $\leq 3 \mathrm{~Gy} /$ fraction and total dose $\geq 60 \mathrm{~Gy}$. Chi-square/ANOVA tests compared clinical/demographic characteristics. Cochran-Armitage test assessed utilization trends over time. MVA computed odds of receiving SBRT. Tests were two-sided with a 0.05 level of significance. Analyses used SAS 9.4 (SAS Institute Inc). This study was waived by institutional review board.

Overall, 1157 men were treated with SBRT and 48,498 with EBRT. Supplementary Table shows clinical/demographic characteristics between groups. Higher SBRT use 
was associated with academic treatment center, more affluent zip code of residence, higher education level, treatment in Northeast US, longer travel distance for treatment, lower T-stage, lower Gleason score, and higher PSA.

SBRT use increased over threefold from 2004 to 2016 ( $p$ $<0.001$ ), which was largely driven by increased utilization in men not receiving (ADT; $0.7 \%$ in 2004 to $8.3 \%$ in 2016, $p<0.001)$ and those with PSA $>20$ as the only HR factor (1.0\% in 2004 to $4.3 \%$ in 2016, $p<0.001$ ); trend graphs are shown in Supplementary Figure.

On MVA, omission of concomitant ADT, clinical stage T12, and Gleason score 6-7 were associated with increased odds of SBRT receipt (Table 1). In addition, the presence of at least one comorbidity, longer travel distance for treatment, and nonMedicaid insurance was also associated with receiving SBRT.

Similar demographic characteristics are associated with receipt of SBRT in HRPCa as other risk groups [1, 2]. In addition, in HRPCa, longer travel distances and medical comorbidities are associated with receiving SBRT, which is concordant with NCCN guidelines encouraging its use when protracted courses create medical/social hardship [3]. Furthermore, we found men treated with SBRT compared to EBRT were more likely to have ADT omitted, lower clinical stage, and lower Gleason score. In fact, the rise in SBRT use for HRPCa has been dominated by two groups: men with PSA $>20$ as the only $\mathrm{HR}$ factor and men not receiving concomitant ADT.

These findings are relevant. First, the subset of HRPCa with PSA > 20 as the only qualifying risk factor has been shown to have lower PCa mortality, similar to those with intermediate risk PCa [7]. Thus, men with more favorable disease characteristics more often received SBRT, and retrospective studies may overestimate the safety of SBRT. Studies demonstrating effectiveness of SBRT for HRPCa, as compared to conventionally fractionated regimens with larger target margins $+/$ - pelvic nodal treatment, should be scrutinized regarding risk stratifying variables (e.g., representative inclusion of stage T3-4 and/or Gleason 8-10) before extrapolating to all HR cases.

Second, SBRT use was significantly associated with omission of ADT, but it remains unclear if ADT was omitted due to the utilization of SBRT, or SBRT was used due the omission of ADT for other reasons, such as patient refusal or comorbidities. Although HYPO-RT-PC [4] provides level one basis for omitting ADT with SBRT in HRPCa with similar 5-year progression-free survival as EBRT, this trial did not permit ADT in either treatment arm and was published well after the timeframe of this study. Results from the ongoing PACE-C trial (NCT01584258), which is a randomized trial comparing hypofractionated EBRT (60 Gy in 20 fractions) plus ADT versus SBRT plus ADT in intermediate and HR PCa, will be informative and potentially validating. Nonetheless, the benefit of concomitant ADT with radiation for HRPCa has been well established [8-10], and underutilization of ADT with SBRT is concerning.

This analysis has limitations. First, currently available NCDB data are only available through 2016, and utilization after 2016 could not be evaluated but would be of interest given more recent publication of HYPO-RT-PC trial and NCCN-endorsement of SBRT for HRPCa. Second, the findings are subject to the inherent biases of its retrospective nature. Third, data regarding details of radiation technique, such as use of CyberKnife or linear accelerator platforms, are unavailable and may be of interest to determine where and how patients are predominantly being treated with SBRT.

In conclusion, SBRT use for HRPCa increased threefold from 2004 to 2016 and will continue to rise given recent guideline support and emerging level one evidence. Favorable disease characteristics and, more concerningly, omission of ADT were associated with receipt of SBRT over EBRT. Randomized trials comparing SBRT with or without ADT for HRPCa are needed before considering omitting ADT.

Funding Research was supported in part by the Biostatistics and Bioinformatics Shared Resource of Winship Cancer Institute of Emory University and NIH/NCI under award number P30CA138292. The content is solely the responsibility of the authors and does not necessarily represent the official views of the National Institutes of Health.

\section{Compliance with ethical standards}

Conflict of interest The authors declare that they have no conflict of interest.

Publisher's note Springer Nature remains neutral with regard to jurisdictional claims in published maps and institutional affiliations.

Open Access This article is licensed under a Creative Commons Attribution 4.0 International License, which permits use, sharing, adaptation, distribution and reproduction in any medium or format, as long as you give appropriate credit to the original author(s) and the source, provide a link to the Creative Commons license, and indicate if changes were made. The images or other third party material in this article are included in the article's Creative Commons license, unless indicated otherwise in a credit line to the material. If material is not included in the article's Creative Commons license and your intended use is not permitted by statutory regulation or exceeds the permitted use, you will need to obtain permission directly from the copyright holder. To view a copy of this license, visit http://creativecommons. org/licenses/by/4.0/.

\section{References}

1. Mahase SS, D'Angelo D, Kang J, Hu JC, Barbieri CE, Nagar H. Trends in the use of stereotactic body radiotherapy for treatment of prostate cancer in the United States. JAMA Netw Open. 2020;3:e1920471.

2. Malouff TD, Stoss WC, Seneviratne DS, Waddle MR, May BC, Buskirk SJ, et al. Current use of stereotactic body radiation therapy for low and intermediate risk prostate cancer: a National 
Cancer Database Analysis. Prostate Cancer Prostatic Dis. 2020;23:349-55.

3. National Comprehensive Cancer Network. Prostate cancer (NCCN Guidelines Version 2.2020). 2020. https://www.nccn.org/ professionals/physician_gls/pdf/prostate.pdf.

4. Widmark A, Gunnlaugsson A, Beckman L, Thellenberg-Karlsson C, Hoyer M, Lagerlund M, et al. Ultra-hypofractionated versus conventionally fractionated radiotherapy for prostate cancer: 5year outcomes of the HYPO-RT-PC randomised, non-inferiority, phase 3 trial. Lancet. 2019;394:385-95.

5. Jackson WC, Silva J, Hartman HE, Dess RT, Kishan AU, Beeler $\mathrm{WH}$, et al. Stereotactic body radiation therapy for localized prostate cancer: a systematic review and meta-analysis of over 6000 patients treated on prospective studies. Int J Radiat Oncol Biol Phys. 2019; 104:778-89.

6. Gonzalez-Motta A, Roach M. Stereotactic body radiation therapy (SBRT) for high-risk prostate cancer: Where are we now? $\mathrm{Pr}$ Radiat Oncol. 2018;8:185-202.
7. Muralidhar V, Chen MH, Reznor G, Moran BJ, Braccioforte MH, Beard CJ, et al. Definition and validation of "Favorable High Risk Prostate Cancer": implications for personalizing treatment of radiation-managed patients. Int $\mathrm{J}$ Radiat Oncol Biol Phys. 2015;93:828-35.

8. Bolla M, Van Tienhoven G, Warde P, Dubois J, Mirimanoff R, Storme G, et al. External irradiation with or without long-term androgen suppression for prostate cancer with high metastatic risk: 10-year results of an EORTC randomised study. Lancet Oncol. 2010;11:1066-73.

9. Pilepich MV, Winter K, Lawton CA, Krisch RE, Wolkov HB, Movsas B, et al. Androgen suppression adjuvant to definitive radiotherapy in prostate carcinoma-long term results of phase III RTOG 85-31. Int J Radiat Oncol Biol Phys. 2005;61:1285-90.

10. Warde P, Mason M, Ding K, Kirkbride P, Brundage M, Cowan R, et al. Combined androgen deprivation therapy and radiation therapy for locally advanced prostate cancer: a randomised, phase 3 trial. Lancet. 2011;378:2104-11. 